\title{
Bordism groups of immersions and classes represented by self-intersections
}

\author{
PETER J ECCLES \\ MARK GRANT
}

\begin{abstract}
A well-known formula of R J Herbert's relates the various homology classes represented by the self-intersection immersions of a self-transverse immersion. We prove a geometrical version of Herbert's formula by considering the self-intersection immersions of a self-transverse immersion up to bordism. This clarifies the geometry lying behind Herbert's formula and leads to a homotopy commutative diagram of Thom complexes. It enables us to generalise the formula to other homology theories. The proof is based on Herbert's but uses the relationship between self-intersections and stable Hopf invariants and the fact that bordism of immersions gives a functor on the category of smooth manifolds and proper immersions.
\end{abstract}

$57 \mathrm{R} 42 ; 57 \mathrm{R} 90,55 \mathrm{~N} 22$

\section{Introduction}

Given a self-transverse immersion $f: M^{n-k} \rightarrow N^{n}$ we define for each integer $r \geq 1$ the $r$-fold self-intersection sets of $f$, in $N$ and $M$ respectively, as

$$
\begin{aligned}
N_{r} & :=\left\{n \in N|| f^{-1}(n) \mid \geq r\right\} \subseteq N, \\
M_{r} & :=f^{-1}\left(N_{r}\right) \subseteq M .
\end{aligned}
$$

(Here and elsewhere the superscript denotes dimension, and all manifolds and maps are assumed smooth, so that for example $N^{n}$ is a smooth $n$-manifold while $N^{(n)}$ is the cartesian product of $n$ copies of $N$.) Using self-transversality of $f$ it can be shown (see Section 4.1) that each of these sets is the image of an immersed $(n-r k)$-manifold; that is, we have immersions

$$
\psi_{r}(f): \Delta_{r}(f)^{n-r k} \rightarrow N^{n}, \quad \mu_{r}(f): \widetilde{\Delta}_{r}(f)^{n-r k} \leftrightarrow \rightarrow M^{n-k},
$$

whose images are $N_{r}$ and $M_{r}$ respectively. An immersion $f: M \rightarrow N$ with $M$ closed represents a class in $H_{*}\left(N ; \mathbb{Z}_{2}\right)$ by evaluating $f_{*}$ on the fundamental class of $M$. It is natural to ask how the homology classes represented by the above self-intersection immersions are related, for different values of $r$. 
In his 1981 thesis [9], R J Herbert answered this question in the following satisfying way. Let $n_{r} \in H^{r k}\left(N ; \mathbb{Z}_{2}\right)$ be the Poincaré dual of the class represented by $\psi_{r}(f)$ (if $N$ is not closed we may use cohomology with compact supports), and let $m_{r} \in$ $H^{(r-1) k}\left(M ; \mathbb{Z}_{2}\right)$ be dual to the class represented by $\mu_{r}(f)$. Let $e \in H^{k}\left(M ; \mathbb{Z}_{2}\right)$ be the Euler class of the normal bundle of $f$.

Theorem 1.1 (Herbert) For $r \geq 1$,

$$
f^{*} n_{r}=m_{r+1}+e \cup m_{r} \in H^{r k}\left(M ; \mathbb{Z}_{2}\right) .
$$

This formula also holds in $H^{*}(M ; \mathbb{Z})$ when $M$ and $N$ are oriented and the codimension $k$ is even. The case $r=1$ was known to Whitney 40 years earlier [28]. Many papers and even chapters in books have been devoted to this formula and its applications (see Biasi and Saeki [3], Kamata [11], Szúcs [20; 23], Ekholm and Szúcs [7] and Takase [24] for recent examples, and Kirby [12, Chapter 6] which treats a special case). An alternative, shorter proof was offered by Felice Ronga [15], see also Braun and Lippner [4], using ideas of Daniel Quillen [14]. However some of the geometric intuition behind Herbert's original proof becomes lost in abstraction.

Our aim here is to generalise Theorem 1.1 by showing it to be true in any homology theory for which the constituent manifolds and normal bundles have an orientation, and to elucidate the simple geometric ideas behind Herbert's proof. To do this we study bordism groups of immersions as contravariant functors on the category of smooth manifolds and proper immersions. This turns out to be the ideal setting, as operations taking the bordism class of $f$ to that of $\psi_{r}(f)$ exist and are fairly well understood (see Koschorke-Sanderson [13] and Vogel [26]) and also the geometric meanings of constructions such as induced maps and products become apparent.

A preliminary announcement of the results in this paper appeared as [6]. The second author was supported by a grant from the United Kingdom Engineering and Physical Sciences Research Council. We are grateful to the referee for helpful comments and suggestions.

\section{Bordism of immersions}

In this section we describe the functor given by bordism of immersions into a given manifold with a given structure on the normal bundle. Our exposition follows closely that of Vogel [26]. More details may be found in Grant [8]. 


\subsection{Definitions}

Let $N^{n}$ be a connected manifold without boundary, and let $\zeta$ be vector bundle of dimension $k$ over a paracompact base space $X$. We consider immersions of closed manifolds into $N$ with a $\zeta$-structure on their normal bundles; that is, triples $\left(M^{n-k}, f, v\right)$ where $M$ is a compact manifold without boundary, $f: M \rightarrow N$ is an immersion, and $v: v(f) \rightarrow \zeta$ is a bundle map from the normal bundle of $f$ which is a vector space isomorphism on each fibre. We may put a bordism relation on such triples in the following way. Two such triples $\left(M_{0}, f_{0}, v_{0}\right)$ and $\left(M_{1}, f_{1}, v_{1}\right)$ are bordant if there exists a triple $\left(W^{n-k+1}, F, V\right)$, where $W$ is a manifold with boundary, $F: W \rightarrow N \times I$ where $I$ is the unit interval $I=[0,1]$, and $V: v(F) \rightarrow \zeta$ is a bundle map, and the following conditions are satisfied:

(1) $\partial W=M_{0} \sqcup M_{1}$;

(2) $F$ is transversal to $\partial(N \times I)=N \times \partial I$;

(3) $F(\partial W) \subseteq N \times \partial I$ and $\left.F\right|_{M_{i}}=f_{i} \times i$, for $i=0,1$;

(4) $\left.v(F)\right|_{M_{i}} \cong v\left(f_{i}\right)$ and $\left.V\right|_{v\left(f_{i}\right)}=v_{i}$.

Here it is understood that ' $=$ ' means 'equals up to diffeomorphism'. The resulting set of bordism classes will be denoted by $\mathcal{I}(N ; \zeta)$, and the class of a triple $(M, f, v)$ will usually be written $[f]$ for brevity. It is clear that the set $\mathcal{I}(N ; \zeta)$ has a commutative monoid structure arising from the disjoint union of immersions, and that the empty immersion provides a zero element. The complete homotopy classification of these monoids may be described (after Wells [27]) as follows.

Theorem 2.1 When $k=\operatorname{dim}(\zeta) \geq 1$,

$$
\mathcal{I}(N ; \zeta) \cong\left[N_{c}, T \zeta\right]_{S} \cong\left[N_{c}, Q T \zeta\right]
$$

When $k=\operatorname{dim}(\zeta)=0$,

$$
\mathcal{I}(N ; \zeta) \cong\left[N_{c}, \bigvee_{r=1}^{\infty}\left(B S_{r}\right)_{c}\right]
$$

Here $T \zeta$ is the Thom space of $\zeta, N_{c}$ is the one-point compactification of $N$, and $Q X$ is the direct limit of the spaces $\Sigma^{\ell} \Omega^{\ell} X$ (as $\ell \rightarrow \infty$ ) for pointed $X$. The space $B S_{r}$ is the classifying space of the symmetric group $S_{r}$.

In the case of positive codimension, Wells proved the above for $N$ the Euclidean space $\mathbb{R}^{n}$ and $\zeta=\gamma_{k}$, the universal $O(k)$-bundle. The result can be deduced for general $N$ and $\zeta$ from Thom's theorem on the bordism of embeddings [25] and the following useful theorem of differential topology due to Rourke and Sanderson [16; 17]. 
Theorem 2.2 (Compression Theorem) Let $g: M^{n-k} \hookrightarrow N^{n} \times \mathbb{R}^{\ell}$ be an embedding with $\ell$ linearly independent normal vector fields, so that $v(g) \cong v \oplus \varepsilon^{\ell}$ for some $k$-dimensional vector bundle $v$. If $k \geq 1$ then $g$ is isotopic to an embedding $g^{\prime}$ such that the composition

$$
M \stackrel{g^{\prime}}{\hookrightarrow} N \times \mathbb{R}^{\ell} \stackrel{p r}{\longrightarrow} N
$$

is an immersion $f: M \leftrightarrow N$. Moreover, $v(f) \cong v$.

When the codimension is zero the Compression Theorem fails. However the result of Theorem 2.1 follows from the observation that a codimension zero immersion of a closed manifold is nothing but a finite covering space. A direct proof covering both cases and using configuration space models has been given by Koschorke and Sanderson [13].

Since $\mathcal{I}(N ; \zeta)$ is isomorphic to a stable homotopy group when $k \geq 1$, it has the structure of an Abelian group. To describe the inverse of an element $[f]$, we note that $\mathcal{I}(N ; \zeta)$ is in bijective correspondence with $\mathcal{I}\left(N \times \mathbb{R} ; \zeta \oplus \varepsilon^{1}\right)$ by the Compression Theorem 2.2, and that an inverse in the latter group may be obtained by composing with a reflection. We refer the reader to Szúcs [20] for more details.

There is also an external product pairing

$$
\mathcal{I}(N ; \zeta) \times \mathcal{I}\left(N^{\prime} ; \zeta^{\prime}\right) \stackrel{\times}{\rightarrow} \mathcal{I}\left(N \times N^{\prime} ; \zeta \times \zeta^{\prime}\right),
$$

given fairly obviously by the cartesian product of representatives. This product corresponds to the smash product of stable homotopy classes under the isomorphism of Theorem 2.1.

\subsection{Functoriality}

Theorem 2.1 shows that $\mathcal{I}(-;-)$ is a homotopy bifunctor, covariant in $\zeta$ and contravariant in $N$. This may be interpreted in terms of the differential topology, as we shall now explain.

Let $\mathcal{C}_{k}$ be the category with objects the $k$-dimensional vector bundles and with morphisms the bundle maps which map fibres isomorphically. Let $\mathcal{D}_{0}$ denote the category with objects the smooth manifolds without boundary and with morphisms the proper immersions. A homotopy in $\mathcal{D}_{0}$ is a regular homotopy (a smooth map $F: M \times I \rightarrow N$ such that at each stage $t \in I$ the map $F(-, t): M \rightarrow N$ is an immersion).

Algebraic $8 \mathcal{G}$ Geometric Topology, Volume 7 (2007) 
Proposition 2.3 (Vogel [26]) Bordism of immersions $\mathcal{I}(-;-)$ gives a homotopy bifunctor from $\mathcal{D}_{0}^{o p} \times \mathcal{C}_{k}$ to the category of commutative monoids, where $\mathcal{C}^{o p}$ denotes the opposite category of $\mathcal{C}$.

Proof It is clear that a bundle map $\eta: \zeta \rightarrow \xi$ induces a map $\eta_{*}: \mathcal{I}(N ; \zeta) \rightarrow \mathcal{I}(N ; \xi)$ so that $\mathcal{I}(N ;-)$ gives a covariant functor from $\mathcal{C}_{k}$ to the category of commutative monoids. Also note that bundle homotopic bundle maps induce the same map of monoids, so that we do indeed have a homotopy functor.

We now describe contravariance in $N$. Given a class $[f]$ in $\mathcal{I}(N ; \zeta)$ and a proper immersion $g$ : $Q^{n-\ell} \rightarrow \rightarrow N^{n}$, we may choose a representative $f^{\prime}$ of $[f]$ which is transverse to $g$ and regularly homotopic to $f$; then the map $\delta$ in the following pullback diagram is an immersion.

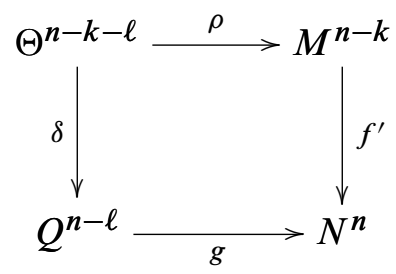

It can be shown (using Theorem 2.2) that $v(\delta) \cong \rho^{*} v\left(f^{\prime}\right) \cong \rho^{*} v(f)$, and so we have a bundle map $\bar{\rho}: v(\delta) \rightarrow v(f)$. The manifold $\Theta$ is closed, since $g$ is proper. This allows us to define the induced map

$$
g^{*}: \mathcal{I}(N ; \zeta) \rightarrow \mathcal{I}(Q ; \zeta)
$$

by sending the class of $(M, f, v)$ to the class of $(\Theta, \delta, v \circ \bar{\rho})$. It is easily checked that this is a well-defined map of monoids, and functoriality is evident. One may also verify that pulling back by regularly homotopic immersions of $Q$ in $N$ results in bordant immersions in $Q$.

\section{Remarks 2.4}

(i) Let $g$ be an immersion and $\eta$ a bundle map. Then the induced maps $g^{*}$ and $\eta_{*}$ are seen to correspond under Theorem 2.1 to composition with the stable homotopy classes of $g_{c}: Q_{c} \rightarrow N_{c}$ and $T \eta: T \zeta \rightarrow T \xi$.

(ii) Functoriality and the diagonal embedding $\triangle: N \rightarrow N \times N$ gives an internal product

$$
\mathcal{I}(N ; \zeta) \times \mathcal{I}\left(N ; \zeta^{\prime}\right) \stackrel{\cup}{\rightarrow} \mathcal{I}\left(N ; \zeta \times \zeta^{\prime}\right)
$$

by setting $[f] \cup\left[f^{\prime}\right]=\triangle^{*}\left([f] \times\left[f^{\prime}\right]\right)$. 


\subsection{Relative groups}

With a little care we may extend the definition of $\mathcal{I}(-; \zeta)$ to the category $\mathcal{D}$ of smooth manifolds with boundary, the morphisms of which are those proper immersions which do not carry interior points to boundary points. Note however that morphisms in $\mathcal{D}$ are not required to preserve boundaries.

Let $N$ have boundary $\partial N$. Now the data are triples $(M, f, v)$ where $M$ is a compact manifold with boundary $\partial M$, and $f: M \leftrightarrow N$ meets $\partial N$ transversally with $f^{-1}(\partial N)=\partial M$. The precise definition of a bordism between such triples involves the idea of a manifold with corners (as described for example by Jänich [10]) and appears in Grant [8]. Call the resulting set of bordism classes $\mathcal{I}(N, \partial N ; \zeta)$.

\section{Relations with cohomology theories}

In this section we shall outline the relation between the bifunctor $\mathcal{I}(-;-)$ and the various generalised (co)homology theories known as (co)bordism (further details may be found in the references). Such theories arise from the various Thom spectra (see for example Stong [18] or Switzer [19]) which are constructed as follows.

Suppose we are given a family $\mathbf{X}=\left\{X_{k}, f_{k}, g_{k}\right\}_{k \geq 0}$, where for each $k$ we have a space $X_{k}$, a fibration $f_{k}: X_{k} \rightarrow B O(k)$ and a map $g_{k}: X_{k} \rightarrow X_{k+1}$ making the following diagram commute.

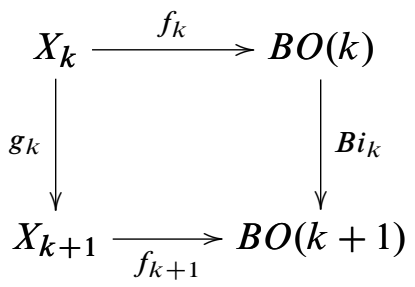

Here $i_{k}: O(k) \hookrightarrow O(k+1)$ is the inclusion homomorphism, and if $\gamma_{k}$ is the universal $O(k)$-bundle over $B O(k)$, we have $\left(B i_{k}\right)^{*} \gamma_{k+1} \cong \gamma_{k} \oplus \varepsilon^{1}$. From these data we get a sequence of bundles $\zeta=\left\{\zeta_{k}:=f_{k}^{*} \gamma_{k}\right\}_{k \geq 0}$, with $\operatorname{dim}\left(\zeta_{k}\right)=k$, and bundle maps $\bar{g}_{k}: \zeta_{k} \oplus \varepsilon^{1} \rightarrow \zeta_{k+1}$. This gives rise to a Thom spectrum $\mathrm{M} \zeta$, with $k$ th space $T \zeta_{k}$, and hence to generalised cohomology and homology theories $M \zeta^{*}$ and $M \zeta_{*}$.

We suppose further that $M \zeta$ is a ring spectrum, the multiplicative structure coming from compatible bundle maps $\zeta_{k} \times \zeta_{\ell} \rightarrow \zeta_{k+\ell}$ inducing maps $T \zeta_{k} \wedge T \zeta_{\ell} \rightarrow T \zeta_{k+\ell}$ for all $k, \ell \geq 0$. This furnishes our (co)bordism theory with cup and cap products.

We shall need some notion of orientability of manifolds with respect to the spectrum M५. 
Definition 3.1 A $\zeta$-manifold is a smooth manifold $N$ together with an equivalence class of $\zeta$-structures on its stable normal bundle. Thus we have an embedding $g: N^{n} \hookrightarrow \mathbb{R}^{n+\ell}$ and a homotopy class of bundle maps $v(g) \rightarrow \zeta_{\ell}$, where $\ell$ is large.

\subsection{Quillen's geometric cobordism and duality}

Let $N^{n}$ be a manifold. It is well known that the bordism theory $\mathrm{M} \zeta_{*}$ has a geometric interpretation, given by Atiyah [1]. Here $M \zeta_{n-k}(N)$ is the set of bordism classes of singular maps $f: M^{n-k} \rightarrow N^{n}$, where $M$ is a closed $\zeta$-manifold. Less well known is Quillen's geometric interpretation of the cobordism group $M \zeta^{k}(N)$ as equivalence classes of $\zeta$-oriented codimension $k$ maps [14].

Definition 3.2 A map of manifolds $f: M^{n-k} \rightarrow N^{n}$ is $\zeta$-orientable if it has a factorisation

$$
M \stackrel{i}{\longrightarrow} E \stackrel{p}{\longrightarrow} N,
$$

where $p: E \rightarrow N$ is an $(r-k)$-dimensional smooth vector bundle over $N$ for some large $r$ (so that $E$ is a $(n-k+r)$-manifold), and $i$ is an embedding with a $\zeta_{r}-$ structure on its normal bundle. A $\zeta$-orientation of $f$ is a class of such factorisations with respect to a suitable equivalence relation.

Quillen puts a cobordism relation on the class of codimension $k \zeta$-oriented maps $f: M \rightarrow N$ with $M$ closed. Then a generalisation of Thom's original proof for the coefficient groups shows that the resulting set of cobordism classes is isomorphic to

$$
M \zeta^{k}(N) \cong \lim _{r \rightarrow \infty}\left[\Sigma^{r} N_{c}, T \zeta_{k+r}\right]
$$

We now suppose that $N$ is a closed $\zeta$-manifold. This gives a Poincaré-Atiyah duality isomorphism $\mathrm{M} \zeta^{k}(N) \cong \mathrm{M} \zeta_{n-k}(N)$ as in [1], which is described geometrically by the identity on representatives. A map $f: M \rightarrow N$ between closed $\zeta$-manifolds has a canonical $\zeta$-orientation, and so represents both a bordism and a cobordism class; these classes are dual to each other.

\section{2 $\mathcal{I}\left(N ; \zeta_{k}\right)$ in this setting}

Now let an element $[f] \in \mathcal{I}\left(N ; \zeta_{k}\right)$ be represented by the triple $\left(M^{n-k}, f, v\right)$. Choose an embedding $f_{1}: M \hookrightarrow \mathbb{R}^{\ell}$, for $\ell$ large. Let $i: M \hookrightarrow N \times \mathbb{R}^{\ell}$ be the embedding given by $i(m)=\left(f(m), f_{1}(m)\right)$ for $m \in M$. This gives a factorisation of $f$ as

$$
M \stackrel{i}{\longrightarrow} N \times \mathbb{R}^{\ell} \stackrel{p r}{\longrightarrow} N,
$$

Algebraic $8 \mathcal{G}$ Geometric Topology, Volume 7 (2007) 
and the sequence of bundle maps

$$
v(i) \cong v(f) \oplus \varepsilon^{\ell} \stackrel{v \oplus 1}{\longrightarrow} \zeta_{k} \oplus \varepsilon^{\ell} \rightarrow \zeta_{k+\ell}
$$

shows that we in fact have a $\zeta$-orientation of $f$. After checking that a bordism between immersions with $\zeta_{k}$-structure gives a cobordism between $\zeta$-oriented maps, we have a well defined map $\Theta_{k}: \mathcal{I}\left(N ; \zeta_{k}\right) \rightarrow \mathrm{M} \zeta^{k}(N)$, which is seen to coincide with the map

$$
\left[\Sigma^{\ell} N_{c}, \Sigma^{\ell} T \zeta_{k}\right] \rightarrow\left[\Sigma^{\ell} N_{c}, T \zeta_{k+\ell}\right]
$$

given by the maps in the spectrum $M \zeta$.

In his paper [14], Quillen also gives the geometric constructions of induced maps, addition and products in the theory $\mathrm{M \zeta}^{*}$, and these are completely analogous to the constructions we gave in the case of immersions in the last section. Using this observation, or by examining the maps in homotopy theory, we see that each $\Theta_{k}$ is a homomorphism of monoids (groups for $k \geq 1$ ), and a natural transformation of functors on the category $\mathcal{D}_{0}$. The multiplicativity is expressed in the following diagram.

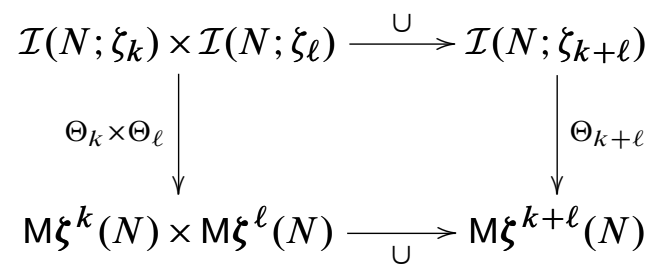

There is also a homomorphism from $\mathcal{I}\left(N ; \zeta_{k}\right)$ to $M \zeta_{n-k}(N)$. This is obtained by regarding an immersion $f: M^{n-k} \rightarrow N^{n}$ as a singular manifold in $N$. Note that $M$ is a $\zeta$-manifold, since its stable normal bundle receives a $\zeta$-structure from that of $v(f)$ and the stable normal bundle of $N$. The image of a class $[f]$ in $M \zeta_{n-k}(N)$ is dual to its image in $M \zeta^{k}(N)$, since both are represented by the map $f: M \rightarrow N$. Finally, suppose we have a Thom class $t: M \zeta \rightarrow \mathrm{A}$, that is, a ring map between ring spectra. Let $A^{*}, A_{*}$ denote the cohomology and homology theories arising from the spectrum A. Then $t$ gives a degree zero multiplicative natural transformation of theories which maps Thom class to Thom class. This also furnishes $N$ with an A-orientation, and hence we have Poincaré Duality with A coefficients and the following commutative diagram.

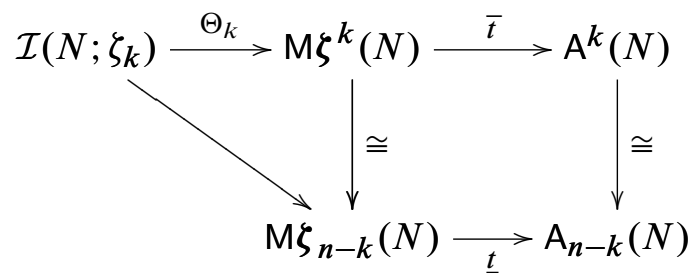

Algebraic $\& \mathcal{G}$ Geometric Topology, Volume 7 (2007) 


\section{Examples 3.3}

(1) The MO spectrum of unoriented bordism, and the so-called 'universal' Thom class $t: \mathrm{MO} \rightarrow \mathrm{H}\left(\mathbb{Z}_{2}\right)$.

(2) The MSO spectrum of oriented bordism and the Thom class $t: \mathrm{MSO} \rightarrow \mathrm{H}(\mathbb{Z})$.

(3) The MU spectrum of complex bordism and the Conner-Floyd map $t: \mathrm{MU} \rightarrow \mathrm{K}$ to complex $K$-theory.

\section{Some operations in the bordism of immersions}

Following Vogel [26] and Koschorke and Sanderson [13], we now introduce operations (natural transformations of set-valued co-functors)

$$
\psi_{r}: \mathcal{I}(N ; \zeta) \rightarrow \mathcal{I}\left(N ; \mathcal{S}_{r}(\zeta)\right)
$$

for each integer $r \geq 0$, where $\mathcal{S}_{r}(\zeta)$ is a bundle to be defined. These operations take the bordism class of an immersion $f: M \leftrightarrow N$ to the bordism class of its $r$-fold self-intersection immersion $\psi_{r}(f): \Delta_{r}(f) \rightarrow N$.

\subsection{Constructions and properties}

Let $f: M^{n-k} \rightarrow \rightarrow N^{n}$ be a self-transverse immersion with a $\zeta$-structure on its normal bundle. Write $f^{(r)} \mid$ for the restriction of $f^{(r)}$ to the open submanifold $F(M ; r) \subset$ $M^{(r)}$ of ordered $r$-tuples of distinct points in $M$. By the self-transversality of $f$, this immersion into $N^{(r)}$ is transverse to the diagonal embedding $\triangle: N \hookrightarrow N^{(r)}$. We form the pullback square.

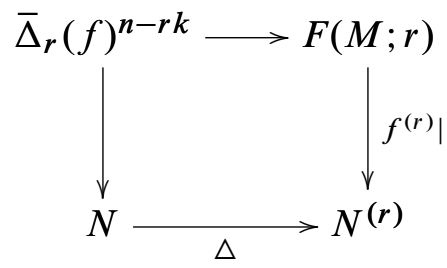

The manifold

$$
\bar{\Delta}_{r}(f)^{n-r k}=\left\{\left(m_{1}, \ldots, m_{r}\right) \in F(M ; r) \mid f\left(m_{1}\right)=\cdots=f\left(m_{r}\right)\right\}
$$

admits free actions of the symmetric groups $S_{r}$ and $S_{r-1}$, by permuting the last $r$ and $(r-1)$ co-ordinates respectively. Call the respective quotient manifolds $\Delta_{r}(f)^{n-r k}$ 
and $\widetilde{\Delta}_{r}(f)^{n-r k}$. We may now define the $r$-fold self-intersection immersion of $f$ in $N$ as

$$
\psi_{r}(f): \Delta_{r}(f)^{n-r k} \leftrightarrow N^{n} ; \quad\left[m_{1}, \ldots, m_{r}\right] \mapsto f\left(m_{1}\right)=\cdots=f\left(m_{r}\right) .
$$

We define the $r$-fold self-intersection immersion of $f$ in $M$ as

$$
\mu_{r}(f): \widetilde{\Delta}_{r}(f)^{n-r k} \leftrightarrow M^{n-k} ; \quad\left(m_{1},\left[m_{2}, \ldots, m_{r}\right]\right) \mapsto m_{1} .
$$

These are indeed immersions of compact manifolds (as shown in Herbert [9]). We shall need to know the structure of their normal bundles.

Let $E S_{r}$ be a contractible space with a free action of $S_{r}$ on the right. For a vector bundle $p: E(\zeta) \rightarrow B(\zeta)$, we define $\mathcal{S}_{r}(\zeta)$ to be the bundle

$$
1 \times_{S_{r}} p^{(r)}: E S_{r} \times_{S_{r}} E(\zeta)^{(r)} \longrightarrow E S_{r} \times_{S_{r}} B(\zeta)^{(r)} .
$$

Here we quotient out by the diagonal action of $S_{r}$ on the product, where $S_{r}$ acts on $X^{(r)}$ on the left by permuting the factors. It is standard that there is a homeomorphism

$$
T \mathcal{S}_{r}(\zeta) \approx D_{r} T \zeta
$$

where $D_{r} X$ is the $r$-adic construction (or equivariant half-smash product) on the pointed space $X$.

It may now be seen fairly easily that a $\zeta$-structure on the normal bundle of $f$ induces a $\mathcal{S}_{r}(\zeta)$ structure on the normal bundle of $\psi_{r}(f)$. The fibre of this normal bundle at a point $\left[m_{1}, \ldots, m_{r}\right] \in \Delta_{r}(f)$ is the unordered direct sum of the fibres of the normal bundle of $f$ at the points $m_{1}, \ldots, m_{r}$. (Similarly one sees that the normal bundle of $\mu_{r}(f)$ has a $\mathcal{S}_{r-1}(\zeta)$-structure). Hence we may define, for $r \geq 1$, an operation

$$
\psi_{r}: \mathcal{I}(N ; \zeta) \rightarrow \mathcal{I}\left(N ; \mathcal{S}_{r}(\zeta)\right)
$$

by setting $\psi_{r}[f]=\left[\psi_{r}\left(f^{\prime}\right)\right]$, where $f^{\prime}$ is a self-transverse representative of $[f]$. This is well defined, since a bordism between self-transverse representatives may be chosen self-transverse, and this defines a bordism between the self-intersection immersions.

Let $\star$ denote the trivial 0 -dimensional bundle over a point, and define $\psi_{0}[f]$ to be $\left[\mathbf{1}_{N}: N \leftrightarrow N\right] \in \mathcal{I}(N ; \star)$. Note that this class acts as an identity for the $U$-product. The operations $\psi_{r}$ for $r \geq 0$ satisfy the following properties.

Proposition 4.1 (Koschorke and Sanderson [13]) Let $[f],[g] \in \mathcal{I}(N ; \zeta)$.

(i) (Naturality) If $h: Q \rightarrow N$, then

$$
h^{*} \psi_{r}[f]=\psi_{r} h^{*}[f] \in \mathcal{I}\left(Q ; \mathcal{S}_{r}(\zeta)\right) .
$$


(ii) $\psi_{1}[f]=[f]$, and $\psi_{r}[f]=0$ for $r>1$ if $[f]$ can be represented by an embedding.

(iii) (Cartan formula)

$$
\psi_{r}([f]+[g])=\sum_{i=0}^{r} \psi_{r-i}[f] \cup \psi_{i}[g] \in \mathcal{I}\left(N ; \mathcal{S}_{r}(\zeta)\right) .
$$

(iv) If $f$ is self-transverse,

$$
\psi_{r}\left[\mu_{2}(f)\right]=\left[\mu_{r+1}(f)\right] \in \mathcal{I}\left(M ; \mathcal{S}_{r}(\zeta)\right)
$$

Proof Properties (i), (ii) and (iv) are direct consequences of the definitions. Property (iii) is slightly less obvious, but the moral is that "If $f \sqcup g$ is self-transverse then an $r$-fold self-intersection of $f \sqcup g$ is the intersection of an $(r-i)$-fold self-intersection of $f$ with an $i$-fold self-intersection of $g$ ". Notice we have made use of bundle maps $\mathcal{S}_{r-i}(\zeta) \times \mathcal{S}_{i}(\zeta) \rightarrow \mathcal{S}_{r}(\zeta)$, which exist due to the product $E S_{r-i} \times E S_{i} \rightarrow E S_{r}$ coming from concatenation of permutations, so that the formula ends up in $\mathcal{I}\left(N ; \mathcal{S}_{r}(\zeta)\right)$.

\subsection{The James-Hopf maps $h^{r}: Q X \rightarrow Q D_{r} X$}

Recall that for a path-connected, pointed space $X$ there is a stable homotopy equivalence

$$
Q X \simeq_{S} \bigvee_{r \geq 1} D_{r} X
$$

the so-called 'Snaith splitting'.

This result rests on the existence of certain James-Hopf maps

$$
h^{r}: Q X \rightarrow Q D_{r} X, \quad r \geq 1,
$$

which may be defined combinatorially using models for the space $Q X$ (see for example Barratt and Eccles [2]). The following theorem, which has been proved by Vogel [26], Szúcs [21; 22] and Koschorke and Sanderson [13], shows how these maps provide a homotopy interpretation of the operations $\psi_{r}$ under the correspondence of Theorem 2.1 .

Theorem 4.2 The following diagram commutes.

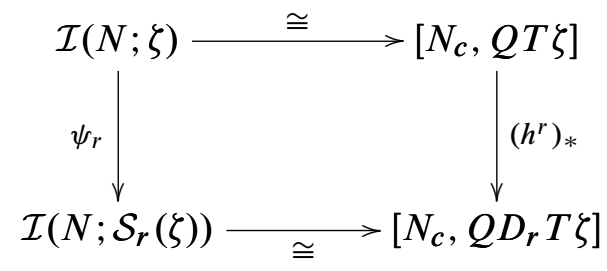

Algebraic $8 \mathcal{G}$ Geometric Topology, Volume 7 (2007) 


\section{Proving Herbert's theorem}

We now prove the analogue of Herbert's Theorem 1.1 in $\mathcal{I}\left(M ; \mathcal{S}_{r}(\zeta)\right)$. Herbert's original proof in [9] was encumbered by the fact that homology classes are represented by singular simplices rather than immersed manifolds. The relative simplicity of our proof stems from the fact that bordism of immersions is in some sense closer to the geometry. Our method is to first prove the double point case, then deduce the general case using the properties of $\psi_{r}$ listed in Proposition 4.1.

\subsection{Proofs}

Let $f: M \leftrightarrow N$ be an immersion of manifolds with $M$ closed, having a $\zeta$-structure on its normal bundle. Hence $f$ represents a class $[f] \in \mathcal{I}(N ; \zeta)$ and the double selfintersection immersion of $f$ in $M, \mu_{2}(f)$, represents a class $\left[\mu_{2}(f)\right] \in \mathcal{I}(M ; \zeta)$. Let $D v(f)$ denote the closed disc bundle of the normal bundle of $f$, and $i: M \hookrightarrow D v(f)$ its zero section which represents a class $[i] \in \mathcal{I}(D v(f) ; \zeta)$.

\section{Proposition 5.1}

$$
f^{*}[f]=\left[\mu_{2}(f)\right]+e \in \mathcal{I}(M ; \zeta),
$$

where $e:=i^{*}[i]$ is the Euler class of $v(f)$.

Proof We may extend $f$ to an immersion $F: D v(f) \rightarrow \rightarrow N$ of the closed disc normal bundle of $f$ which is injective on the fibres. This immersion $F$ does not represent a bordism class, since it does not preserve boundaries. However, it is a morphism in the domain category $\mathcal{D}$ (see Section 2.3), and since $f=F \circ i$, we have

$$
f^{*}[f]=i^{*} F^{*}[f]
$$

by functoriality. So we first give an explicit immersion which represents $F^{*}[f] \in$ $\mathcal{I}(D v(f), \partial D v(f) ; \zeta)$.

Since $F$ has codimension zero, it is automatically transverse to $f$. The pullback construction of Proposition 2.3 gives rise to a manifold

$$
\Theta=\{(v, m) \in D v(f) \times M \mid F(v)=f(m)\},
$$

and an immersion $\delta$ : $\Theta \rightarrow \rightarrow D v(f)$ with $\delta(v, m)=v$. Having insisted that $F$ be injective on fibres, we can partition $\Theta$ as a disjoint union $\Theta=\Theta_{0} \sqcup \Theta_{1}$, where $\Theta_{0}=\{(i(m), m)\}$, and

$$
\Theta_{1}=\left\{(v, m) \mid v \in v(f)_{m_{1}}, m_{1} \neq m\right\} .
$$


Clearly $\Theta_{0}=M$ and the restriction of $\delta$ to $\Theta_{0}$ is identified as $i: M \hookrightarrow D v(f)$. Putting $g:=\left.\delta\right|_{\Theta_{1}}: \Theta_{1} \uparrow D v(f)$, we have

$$
F^{*}[f]=[i]+[g] \in \mathcal{I}(D v(f), \partial D v(f) ; \zeta) .
$$

Applying $i^{*}$ now gives

$$
f^{*}[f]=e+i^{*}[g]
$$

Now we may assume that $f$ is self-transverse. This implies that $i$ is transverse to $g$, making it easy to check that the pullback of $g$ by $i$ is indeed the immersion $\mu_{2}(f): \widetilde{\Delta}_{2}(f) \leftrightarrow M$.

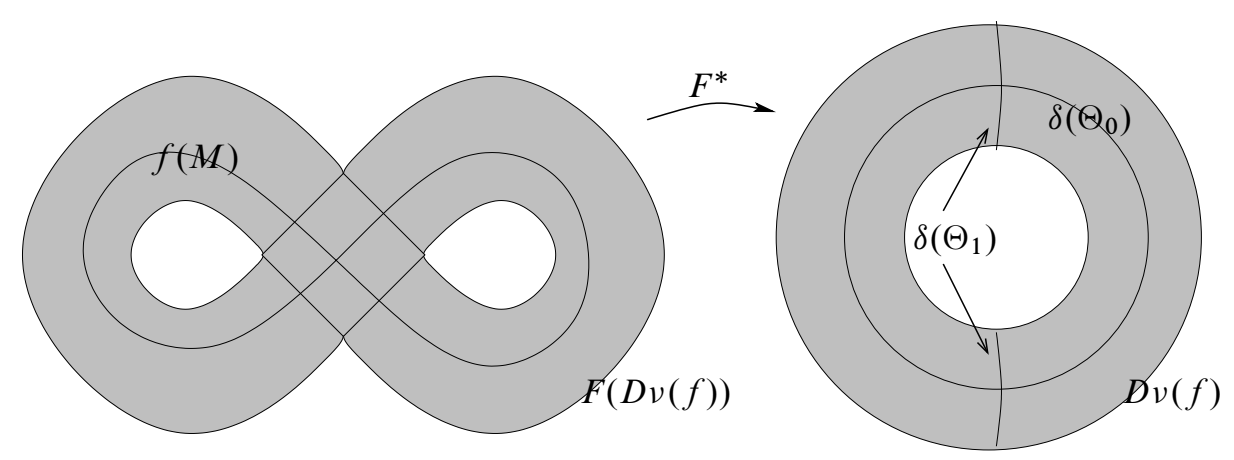

Figure 1: Illustrating Proposition 5.1 for the figure eight immersion.

Example 5.2 Figure 1 depicts the self-transverse immersion $f: S^{1} \rightarrow S^{2}$ given by $f\left(e^{i \theta}\right)=(\cos \theta, \sin 2 \theta)$ with image a figure eight (here we regard $S^{2}$ as the compactified plane). Also shown is the image of an immersion $F: D v(f) \rightarrow S^{2}$ of the (trivial) normal bundle. When we pull back $f$ by $F$ we obtain an immersion $\delta: \Theta \hookrightarrow \rightarrow D v(f)$ whose image is shown on the right. This example clearly shows $\delta\left(\Theta_{0}\right)$ as the image of the zero section $i: M \hookrightarrow D v(f)$, and the intersection of $i$ with $\delta\left(\Theta_{1}\right)$ as the double point set of $f$ in $M$.

Theorem 5.3 For $r \geq 1$,

$$
f^{*}\left[\psi_{r}(f)\right]=\left[\mu_{r+1}(f)\right]+e \cup\left[\mu_{r}(f)\right] \in \mathcal{I}\left(M ; \mathcal{S}_{r}(\zeta)\right)
$$


Proof This follows from the double point case above using Proposition 4.1. The argument is as follows:

$$
\begin{aligned}
f^{*}\left[\psi_{r}(f)\right] & =\psi_{r} f^{*}[f] & & \text { by Proposition 4.1(i) } \\
& =\psi_{r}\left(\left[\mu_{2}(f)\right]+e\right) & & \text { by Proposition 5.1 } \\
& =\sum_{i=0}^{r} \psi_{r-i}\left[\mu_{2}(f)\right] \cup \psi_{i}[e] & & \text { by Proposition 4.1(iii) } \\
& =\psi_{r}\left[\mu_{2}(f)\right]+\psi_{r-1}\left[\mu_{2}(f)\right] \cup e & & \text { by Proposition 4.1(ii) } \\
& =\left[\mu_{r+1}(f)\right]+e \cup\left[\mu_{r}(f)\right] & & \text { by Proposition 4.1(iv). }
\end{aligned}
$$

This completes the proof.

Using the diagram at the end of Section 3, immersions represent classes in certain cohomology theories according to their structure, and we may deduce Herbert's theorem in these theories. Let $\mathrm{M} \zeta$ be the Thom spectrum with $k$ th space $T \zeta_{k}$, and let $f: M \leftrightarrow N$ have a $\zeta_{k}$-structure on its normal bundle. We remark without proof that our Euler class $e:=i^{*}[i]$ maps to the cobordism Euler class of $v(f)$ under the homomorphism $\Theta_{k}: \mathcal{I}\left(M ; \zeta_{k}\right) \rightarrow M \zeta^{k}(M)$ (compare the definition in Quillen [14, Section 2]). Suppose there is a bundle map from $\mathcal{S}_{r}\left(\zeta_{k}\right)$ to $\zeta_{r k}$.

Corollary 5.4 In the above situation Herbert's formula holds in $\mathcal{I}\left(M ; \zeta_{r k}\right)$ and hence in $M \zeta^{r k}$.

\section{Examples 5.5}

(i) The MO spectrum. Let $\gamma_{k}$ be the universal $O(k)$ bundle; then there is a classifying bundle map $\mathcal{S}_{r}\left(\gamma_{k}\right) \rightarrow \gamma_{r k}$ for every $r \geq 1$, and hence Theorem 5.3 is true also in $\mathrm{MO}^{*}$ and $\mathrm{H}\left(\mathbb{Z}_{2}\right)^{*}$. This recovers Theorem 1.1.

(ii) The MSO spectrum. Let $\tilde{\gamma}_{k}$ be the universal $S O(k)$ bundle. When $k$ is even, the bundle $\mathcal{S}_{r}\left(\tilde{\gamma}_{k}\right)$ is orientable, so admits a map to $\tilde{\gamma}_{r k}$. Hence we deduce Theorem 5.3 in the theories $\mathrm{MSO}^{*}$ and $\mathrm{H}(\mathbb{Z})$ when the normal bundle of $f$ is orientable and of even dimension.

(iii) The MU spectrum. Let $\gamma_{j}^{U}$ be the universal $U(j)$ bundle (of real dimension $2 j$ ). The bundle $\mathcal{S}_{r}\left(\gamma_{j}^{U}\right)$ has a complex structure constructed from that of $\gamma_{j}^{U}$. Hence if $f$ is an immersion of even codimension with a complex structure on its normal bundle, we obtain Theorem 5.3 in the theories $\mathrm{MU}^{*}$ and $\mathrm{K}^{*}$. 


\subsection{Homotopy of Thom spaces}

By virtue of Theorem 2.1, bordism classes of immersions are represented by stable homotopy classes of maps into Thom spaces. We may therefore translate the geometric result Theorem 5.3 into a homotopy commutative diagram of representing maps. Write $f: N_{c} \rightarrow Q T \zeta$ for the stable adjoint of a map representing the class $[f] \in \mathcal{I}(N ; \zeta)$. By Section 4.2 and the remarks following Proposition 2.3, the class $f^{*} \psi_{r}[f]$ is represented by the composition

$$
M_{c} \stackrel{f_{c}}{\longrightarrow} N_{c} \stackrel{\stackrel{f}{\longrightarrow}}{\longrightarrow} Q T \zeta \stackrel{h^{r}}{\longrightarrow} Q D_{r} T \zeta .
$$

Similarly, Proposition 4.1(iv) says that $\underline{\mu_{r+1}(f)}$ is the composition

$$
M_{c} \stackrel{\mu_{2}(f)}{\longrightarrow} Q T \zeta \stackrel{h^{r}}{\longrightarrow} Q D_{r} T \zeta .
$$

The Euler class $e \in \mathcal{I}(M ; \zeta)$ is represented by a map $\underline{e}: M_{c} \rightarrow Q T \zeta$.

Corollary 5.6 The following diagram commutes up to homotopy.

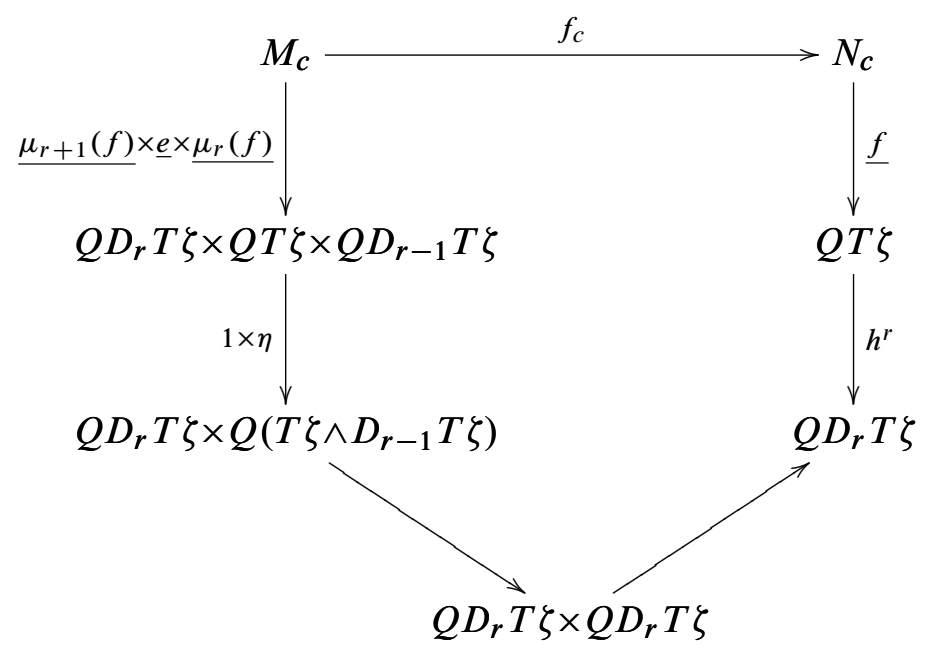

The map $\eta$ is a special case of the smash product pairing $Q X \times Q Y \rightarrow Q(X \wedge Y)$ (see Caruso, Cohen, May and Taylor [5]) defined for pointed spaces $X$ and $Y$ by passage to the limit over the smash product maps $\wedge: \Omega^{k} \Sigma^{k} X \times \Omega^{\ell} \Sigma^{\ell} Y \rightarrow \Omega^{k+\ell} \Sigma^{k+\ell} X \wedge Y$. The unlabelled map on the left is $1 \times Q \xi$, where $\xi: T \zeta \wedge D_{r-1} T \zeta \rightarrow D_{r} T \zeta$ is a standard map (see the proof of Proposition 4.1). The unlabelled map on the right is the loop product, which corresponds to addition in the bordism group. 


\section{References}

[1] MF Atiyah, Bordism and cobordism, Proc. Cambridge Philos. Soc. 57 (1961) 200-208 MR0126856

[2] M G Barratt, P J Eccles, $\Gamma^{+}$-structures III: The stable structure of $\Omega^{\infty} \Sigma^{\infty}$ A, Topology 13 (1974) 199-207 MR0348739

[3] C Biasi, O Saeki, On the Betti number of the image of a codimension- $k$ immersion with normal crossings, Proc. Amer. Math. Soc. 123 (1995) 3549-3554 MR1273476

[4] G Braun, G Lippner, Characteristic numbers of multiple-point manifolds, Bull. London Math. Soc. 38 (2006) 667-678 MR2250759

[5] J Caruso, F R Cohen, J P May, L R Taylor, James maps, Segal maps, and the KahnPriddy theorem, Trans. Amer. Math. Soc. 281 (1984) 243-283 MR719669

[6] P J Eccles, M Grant, Bordism classes represented by multiple point manifolds of immersed manifolds, Tr. Mat. Inst. Steklova 252 (2006) 55-60 MR2255968

[7] T Ekholm, A Szúcs, Geometric formulas for Smale invariants of codimension two immersions, Topology 42 (2003) 171-196 MR1928649

[8] M Grant, Bordism of immersions, $\mathrm{PhD}$ thesis, University of Manchester (2006)

[9] R J Herbert, Multiple points of immersed manifolds, Mem. Amer. Math. Soc. 34 (1981) xiv+60 MR634340

[10] K Jänich, On the classification of $O(n)$-manifolds, Math. Ann. 176 (1968) 53-76 MR0226674

[11] M Kamata, On multiple points of a self-transverse immersion, Kyushu J. Math. 50 (1996) 275-283 MR1447922

[12] R C Kirby, The topology of 4-manifolds, Lecture Notes in Mathematics 1374, Springer, Berlin (1989) MR1001966

[13] U Koschorke, B J Sanderson, Self-intersections and higher Hopf invariants, Topology 17 (1978) 283-290 MR508891

[14] D Quillen, Elementary proofs of some results of cobordism theory using Steenrod operations, Advances in Math. 7 (1971) 29-56 (1971) MR0290382

[15] F Ronga, On multiple points of smooth immersions, Comment. Math. Helv. 55 (1980) 521-527 MR604710

[16] CP Rourke, B J Sanderson, The compression theorem I, Geom. Topol. 5 (2001) 399-429

[17] CP Rourke, B J Sanderson, The compression theorem III: Applications, Algebr. Geom. Topol. 3 (2003) 857-872 MR2012956

[18] R E Stong, Notes on cobordism theory, Mathematical notes, Princeton University Press, Princeton, N.J. (1968) MR0248858 
[19] R M Switzer, Algebraic topology-homotopy and homology, Die Grundlehren der mathematischen Wissenschaften 212, Springer, New York (1975) MR0385836

[20] A Szúcs, Cobordism of singular maps arXiv:math.GT/0612152

[21] A Szúcs, Cobordism groups of l-immersions I: (A) Homotopy representability of the cobordism group of immersions with a given multiplicity of self-intersections, Acta Math. Acad. Sci. Hungar. 27 (1976) 343-358 MR0440557 (Russian)

[22] A Szúcs, Cobordism groups of l-immersions II, Acta Math. Acad. Sci. Hungar. 28 (1976) 93-102 MR0440558 (Russian)

[23] A Szúcs, On the multiple points of immersions in Euclidean spaces, Proc. Amer. Math. Soc. 126 (1998) 1873-1882 MR1451830

[24] M Takase, A geometric formula for Haefliger knots, Topology 43 (2004) 1425-1447 MR2081431

[25] R Thom, Quelques propriétés globales des variétés différentiables, Comment. Math. Helv. 28 (1954) 17-86 MR0061823

[26] P Vogel, Cobordisme d'immersions, Ann. Sci. École Norm. Sup. (4) 7 (1974) 317-357 (1975) MR0372878

[27] R Wells, Cobordism groups of immersions, Topology 5 (1966) 281-294 MR0196760

[28] H Whitney, On the topology of differentiable manifolds, from: "Lectures in Topology", University of Michigan Press, Ann Arbor, Mich. (1941) 101-141 MR0005300

School of Mathematics, University of Manchester

Oxford Road, Manchester M13 9PL, UK

Department of Mathematical Sciences, Durham DH1 3LE, UK

pjeccles@manchester.ac.uk, mark.grant@durham.ac.uk

http://www.maths.manchester.ac.uk/ peter/, http://maths.dur.ac.uk/

$\sim \mathrm{dma} 1 \mathrm{mg} /$

Received: 20 December $2006 \quad$ Revised: 30 March 2007 